\title{
Biorelevant Dissolution: Methodology and Application in Drug Development
}

\author{
Qingxi Wang ${ }^{1}$, Nikoletta Fotaki ${ }^{2}$, and Yun $\mathrm{Mao}^{3}$ \\ ${ }^{1}$ Analytical Development and Commercialization, Merck and Co, Inc., West Point, PA 19486, USA \\ ${ }^{2}$ Department of Pharmacy and Pharmacology, University of Bath, Bath, UK \\ ${ }^{3}$ Pharmaceutical R\&D, Merck and Co, Inc., West Point, PA 19486, USA
}

\section{INTRODUCTION}

D ssolution testing can play an important role in several areas for drug products as a quality control tool to monitor batch-to-batch consistency of drug release from a dosage form and as an in vitro surrogate for in vivo performance that can guide formulation development and ascertain the need for bioequivalence tests. The possibility of substituting dissolution tests for clinical studies has been revealed by the development of the Biopharmaceutics Classification System, and dissolution tests that can predict the in vivo performance of drug products (usually called "biorelevant" dissolution tests) could serve this purpose $(1,2)$. In terms of media and hydrodynamics, biorelevant dissolution testing should provide a baseline for drug and dosage-form performance and should be used to guide formulation development, to identify food effects on the dissolution and bioavailability of orally administered drugs, and to identify solubility limitations and stability issues. The importance of the development of predictive dissolution testing is increased by the fact that the majority of drugs currently in development are poorly soluble drugs and by the challenges for new dosage-form approaches.

\section{METHODOLOGY OF BIORELEVANT DISSOLUTION TESTING}

Dissolution can be affected by drug substance factors (solubility, permeability, dissolution rate), dosage form factors (dissolution characteristics, manufacturing processes), and the methods used for its assessment (apparatus, method, dissolution medium). To develop a biorelevant dissolution test for oral dosage forms, the physiological conditions in the gastrointestinal (GI) tract that can affect drug dissolution (Table 1) should be taken into consideration according to the properties of the drug and dosage form (1). These conditions include the properties of Gl fluids (composition, volume, $\mathrm{pH}$ ), gastric emptying (especially for nondisintegrating systems), intestinal transit, Gl motility and hydrodynamic patterns, Gl enzymes, and the presence or absence of food. Selection of appropriate in vitro conditions (media and hydrodynamics) that simulate the in vivo conditions can lead to the generation of successful in vitro-in vivo correlations (IVIVC) or in vitro-in vivo relationship (IVIVR).

${ }^{3}$ Corresponding author.
Table 1. In Vitro and In Vivo Dissolution Parameters

\begin{tabular}{|c|c|c|}
\hline Parameter & In Vitro Dissolution & In Vivo Dissolution \\
\hline Media & $\begin{array}{l}\text { Compendial media } \\
\text { USP media } \\
\text { Biorelevant media }\end{array}$ & Gastrointestinal fluids \\
\hline Volume & $\begin{array}{l}\text { Variable according to } \\
\text { apparatus used and } \\
\text { simulated condition } \\
\text { (fasted or fed state) }\end{array}$ & $\begin{array}{l}\text { Variable according to } \\
\text { condition (fasted or fed } \\
\text { state) }\end{array}$ \\
\hline Duration & $\begin{array}{l}\text { Variable according to } \\
\text { apparatus used, dosage } \\
\text { form and simulated } \\
\text { condition (fasted or fed } \\
\text { state) }\end{array}$ & $\begin{array}{l}\text { Variable according to } \\
\text { dosage form and } \\
\text { condition (fasted or fed } \\
\text { state) }\end{array}$ \\
\hline Hydrodynamics & USP Apparatus 1, 2, 3, 4 & Gastrointestinal motility \\
\hline Location & Constant* & Variation with time \\
\hline Amount of drug & $\begin{array}{l}\text { Constant in closed systems } \\
\text { Decreases in open system }\end{array}$ & $\begin{array}{l}\text { Decreases as drug is } \\
\text { absorbed }\end{array}$ \\
\hline
\end{tabular}

* Unless media change occurs (i.e., USP Apparatus 3 and 4)

\section{Medium Selection}

Unlike compendial media (conventional buffers, USP media), biorelevant media should represent the gastric and intestinal environment in fasted and fed states $(1,3,4)$. In these media, several properties such as $\mathrm{pH}$, the presence of bile, the buffer capacity, and the surface tension of the $\mathrm{Gl}$ fluids are taken into consideration. Bile salts and phospholipids may have a significant effect on the in vivo dissolution and transport in the small intestine of poorly soluble drug substances. For cases in which lipid-based formulations were characterized, lipolysis could be accounted for by the addition of lipolytic degradation products to the dissolution media to simulate the fed state, because they play an important role in the solubilization capacity of the medium (5).

\section{Hydrodynamics}

In in vitro experiments, hydrodynamics reflects the design of the apparatus; the agitation intensity; the flow, volume, and viscosity of the medium; and practical issues such as the position of the dosage form during the experiment. The choice of the most appropriate hydrodynamics is essential for the development of a biorelevant dissolution method. 
Generally, four basic types of dissolution apparatus are used in the development of biorelevant dissolution testing:

- Rotating basket (USP Apparatus 1)

- Rotating paddle (USP Apparatus 2)

- Reciprocating cylinder Bio-Dis (USP Apparatus 3)

- Flow-through cell (USP Apparatus 4) (closed or open system)

Modifications of these apparatus and new apparatus are also proposed in specific cases, such as the dissolution stress-test device for dosage forms that show sensitivity to physical stress events during gastrointestinal transit (6). USP Apparatus 1 and 2 (referred to as closed systems) are the most used because they are relatively simple and robust and are adequately standardized. They are usually the first choice for solid dosage forms and immediate- and extended-release formulations, but they are not appropriate for all drugs and dosage forms. Problems with homogeneity in the vessel, dispersion in the vessel with areas of high concentration, or agglomeration can appear. Paddle and basket speeds of 50-100 rpm and 50-150 rpm, respectively, are usually used for the development of $\operatorname{IVIVCs}(7,8)$; investigation of lower agitation speeds has been proposed for better representation of in vivo hydrodynamics $(9,10)$. USP Apparatus 3 and 4 are appropriate for drugs and dosage forms where dissolution at multiple $\mathrm{pH}$ levels or sequential changes of media are needed to mimic in vivo dissolution (i.e., controlledrelease dosage forms). For Apparatus 3, dip rates of 10-15 dpm have been proposed for experiments under fasted-state conditions (11). Apparatus 4 offers several cell types and can be particularly useful for specific drug dosage forms (i.e., multiparticulate dosage forms, suspensions). It can operate as either an open or closed system, which is important for poorly soluble drugs. Duration of exposure to the various media and corresponding flow rates should be designed to achieve a balance between the Gl fluid volumes and the physiological residence times in the Gl lumen. Flow rates between 4 and $8 \mathrm{~mL} / \mathrm{min}$ have been proposed for experiments that simulate fasted-and fed-state conditions (12). Moreover, agglomeration in USP Apparatus 4 can be avoided with the use of glass beads in the cell. Recently, it has been shown that with biorelevant dissolution experiments, the hydrodynamics of USP Apparatus 2, 3, and 4 may all be adequate as a starting point for generating IVIVCs for monolithic dosage forms in the fasted state (13).

\section{Drug Substance and Dosage Form Considerations}

For IR dosage forms containing BCS Class I or III compounds, simplified dissolution tests can be used. Dissolution may have little value for rapidly dissolving IR dosage forms; a disintegration test can predict dissolution behavior because disintegration of the dosage form is the rate-limiting step to dissolution. For IR dosage forms containing BCS Class II or IV compounds, drug solubilization and formulation properties have a substantial effect on in vitro and in vivo dissolution. In vitro dissolution profiles in biorelevant tests (biorelevant media in combination with biorelevant hydrodynamics according to the formulation properties) should be evaluated during development, and an IVIVC or IVIVR can be established between the in vitro dissolution and in vivo performance. For lipid-based formulations, biorelevant tests with the addition of lipolytic products are proposed. For MR solid oral dosage forms, dissolution will likely be a critical quality attribute linked to drug product performance. In vivo drug release from these formulations occurs according to a specific predefined delivery pattern, and environmental factors should not influence the release. Oral bioavailability is limited by intralumenal release, and the selection of an appropriate biorelevant dissolution test can lead to prediction of in vivo performance. The use of a $\mathrm{pH}$ gradient and sequential changes of media (use of USP Apparatus 3 and 4) can be valuable during drug development to expose the dosage forms to the different conditions across the Gl tract. The biorelevant dissolution test should be designed according to the dosage-form release pattern and the simulated fasted- or fed-state conditions.

Biorelevant dissolution testing was proved valuable in predicting both the in vivo behavior of lipophilic, poorly water-soluble drugs $(14,15,16)$ and the absorption of BCS Class III compounds in ER formulations when combined with permeability data compared with in vivo data from an inappropriate animal model (e.g., canine model for BCS Class III compounds) (17). More research is needed for realistic representation of in vivo hydrodynamics and in vitro simulation of other conditions that can affect drug absorption (e.g., drug decomposition, enterohepatic recirculation). Research on a combination of biorelevant dissolution tests with biorelevant permeability tests could be valuable for developing predictive tests for BCS Class III and IV compounds.

\section{BIORELEVANT DISSOLUTION IN PRE-CLINICAL DEVELOPMENT}

During preclinical development, candidates are selected and formulated to assess their safety and tolerability. Adequate bioavailability is a prerequisite, and poorly water-soluble compounds present major challenges $(18,19)$. Biorelevant dissolution plays an important role in the selection of appropriate drug candidates and formulations for development. During preclinical development, biorelevant dissolution can be of value in:

- Selecting appropriate drug substance phases and forms for formulation development.

- Developing toxicology formulations with adequate bioavailability to evaluate drug safety and tolerability in animals. 
- Developing formulations with adequate bioavailability to evaluate drug safety and tolerability in Phase I studies.

- Guiding quality control method development to evaluate batch-to-batch consistency.

API properties can significantly impact formulation dissolution and bioavailability. Consideration of chemical and physical stability, processibility, and bioavailability needs to be balanced in API phase selection. Salt formation and particle size reduction are mechanisms that can enhance dissolution and bioavailability (20). Biorelevant dissolution of various drug substance phases can facilitate the selection of appropriate salt forms, polymorphs, and particle sizes. At early stages, a simple and universal dissolution test using a biorelevant dissolution medium should be used. The amount of drug substance is based on the compound potency and projected human doses. The primary goal of drug substance dissolution is to examine the impact of drug substance attributes on the rate and extent of dissolution in biorelevant media (21). The salt forms of basic compounds can have higher solubilities in gastric $\mathrm{pH}$, and precipitation to neutral forms can occur at intestinal $\mathrm{pH}$. The salt forms of weakly acidic compounds can disproportionate to the neutral forms at gastric $\mathrm{pH}$ and resolubilize in intestinal $\mathrm{pH}$. Kinetics of drug dissolution, precipitation, and resolubilization can be monitored either in situ or offline. For neutral compounds, reducing particle size can lead to dissolution enhancement. However, very small particles can form large aggregates, which leads to slow dissolution. Sonicating drug suspensions or dispersing drug with excipients can break agglomerates before biorelevant dissolutions.

Understanding API dissolution in biorelevant media also builds a solid foundation for further formulation development. The goal of dosage form development is to solubilize the maximum amount of API and maintain it in solution for the longest period of time within the absorption window. Biorelevant dissolution helps to assess the challenges of dissolving all the doses required in the Gl tract (22). The higher the challenges are, the higher the bioperformance risk and the greater the development efforts that are likely needed. Biorelevant dissolution also informs where the drug will be most solubilized in the Gl tract and guides pharmaceutical scientists in achieving the desired bioavailability with different formulations. For example, using solid dispersions, predissolving the drug in lipids, or reducing particle size can enhance the apparent solubility. Use of excipients such as surfactants, water-soluble fillers, and superdisintegrants leads to faster dissolution rates. Polymers and antinucleation agents can be used to maintain salts in solution and delay precipitation to neutral forms.

The dosage form for toxicology studies is typically a solution or suspension. The dose range is often wide to evaluate safety and tolerability in animals. For lipids or aqueous-based solution formulations, the key challenge is to maintain the API in solution when the formulation is dissolved or diluted with biorelevant media. For suspension-based formulations, a rank order can be obtained based on the rate and extent of dissolution. It is important to note that there are anatomical and physiological differences, such as $\mathrm{pH}$, bile salt type, and transition time, between animals and humans. Simulated animal fluids can better predict in vivo performance $(23,24)$.

The target product profiles including dose strength, desired in vivo pharmacokinetic (PK) profile, and food-effect restrictions, need to be considered in Phase I dosage form development. Many dosage form options are available for poorly water-soluble compounds. The challenge is to select the most appropriate dosage form with limited development time and limited drug supply. Biorelevant dissolution, coupled with absorption modeling, serves as an efficient and effective in vitro dosage-form screening tool. Human doses, API properties, BCS classification, and dosage-form approaches need to be considered for biorelevant dissolution. Simple USP Apparatus 1 and 2 with biorelevant medium can be used as the starting model. USP Apparatus 3 and 4 can also be explored when maintaining sink conditions or evaluating multiple $\mathrm{pH}$ conditions are important. The solution or suspension toxicology formulations can serve as benchmarks for Phase I formulations and can be evaluated during biorelevant dissolution. Computer modeling using biorelevant dissolution and permeation data can predict in vivo performance risk (25). The lead formulation can be evaluated further in animals if the in vivo performance risk is deemed high. IVIVC and IVIVR can be explored when multiple formulations are evaluated in animals.

\section{BIORELEVANT DISSOLUTION IN PHASE I CLINICAL DEVELOPMENT}

Human PK data is available after Phase I clinical development. The plasma concentration profile, variability, dose proportionality, and food effect provide important feedback on Phase I formulation. Deconvolution of the plasma concentration data after oral administration creates the in vivo dissolution profile. In vitro dissolution, if biorelevant, should correlate with the in vivo dissolution. For example, if human exposure reaches a plateau at a certain dose, a solubility limited in vivo dissolution should be observed. If positive or negative food effects are observed, the difference between fed-and fasted-state dissolution should trend with in vivo data (26). If needed, the dissolution apparatus, medium $\mathrm{pH}$, and hydrodynamics should be adjusted to match the in vivo dissolution. IVIVC and IVIVR can be explored to improve formulation performance.

For Phase I clinical studies, a quality control (QC) method is often used to monitor the formulation batch-to-batch consistency and stability. Simple aqueous 


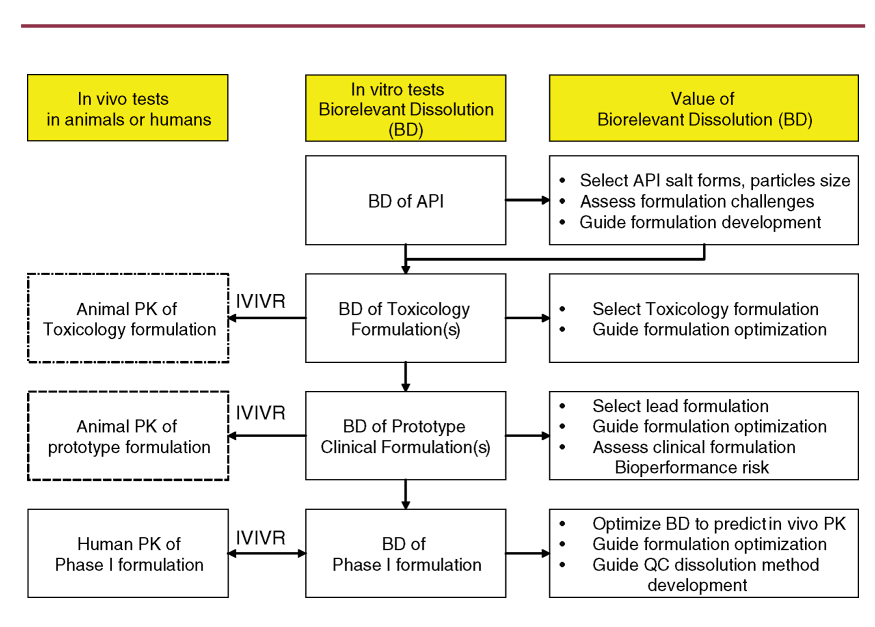

Figure 1. Biorelevant dissolution in early phase of drug product development.

media or surfactant media with adequate sink conditions are selected. During early development, the batch-tobatch consistency is less critical because few batches are made for Phase I clinical studies. Biorelevant dissolution is arguably a more meaningful dissolution test at this stage. A disintegration test can serve as a surrogate to monitor batch-to-batch consistency. As the program moves to later stages of development, simple and robust quality control dissolution becomes more important. Ideally, the QC dissolution method should also be biorelevant and reflect in vivo dissolution. The values of biorelevant dissolution in early phase development and opportunities for IVIVC/R are summarized in Figure 1.

\section{BIORELEVANT DISSOLUTION IN PHASE II CLINICAL DEVELOPMENT}

During preclinical and Phase I stages of drug development, a suitable biorelevant dissolution method is developed to probe potential food effects and rank-order IVIVC or IVIVR largely based on animal data and simulation or modeling tools to guide formulation development. At Phase II clinical development, however, a preliminary market formulation is defined and tested in Phase II and III studies. Human PK and PD data, which can be used to correlate with data from biorelevant dissolution in the development of IVIVC, are frequently collected intentionally or unintentionally. Herein, biorelevant dissolution, as validated by animal data from preclinical and Phase I development, presents the most likely opportunity for IVIVC/R. This also can be facilitated through modeling and simulation. In general, the value of biorelevant dissolution can be used in the following critical activities:

- Bridging Phase I and Phase II (i.e., preliminary market) formulations.

- Developing a preliminary market formulation.

- Developing a design space and linkage between design space and target product profile.

- Defining Critical Quality Attributes (CQA) and Critical Process Parameters (CPP).

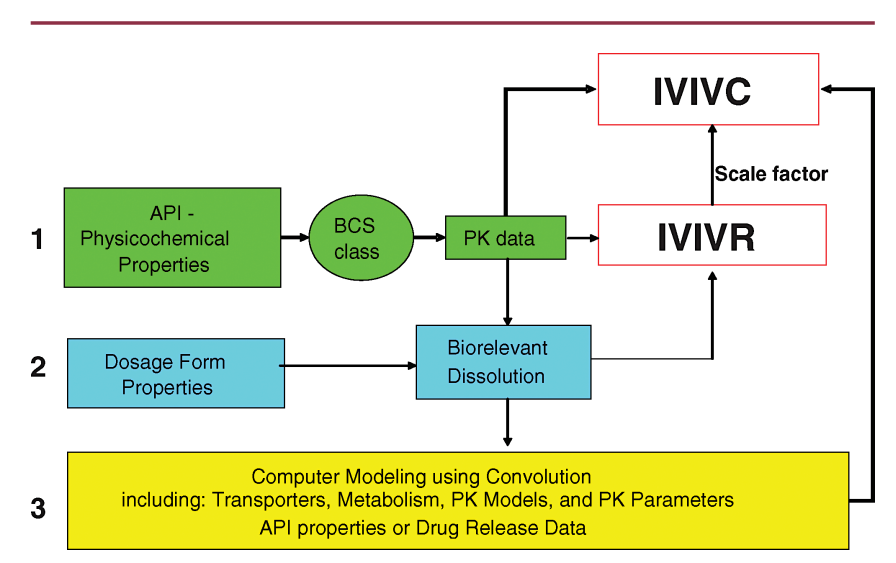

Figure 2. Biorelevant dissolution in development of IVIVC and IVIVR.

- Serving as a quality control tool for batch release and batch-to-batch consistency.

- Monitoring collective changes in stability.

Establishment of an IVIVC or IVIVR is the prerequisite for any dissolution method to be biorelevant. Figure 2 provides a roadmap for developing an IVIVC or IVIVR, which also represents the process of developing or validating a biorelevant dissolution method. A basic relationship might be found between API properties and PK data (level 1). This can be in the form of a rank order or can be modeled mathematically. In the next level (level 2), deconvolution of PK data after oral administration might be used to establish an IVIVC or IVIVR. This can be achieved by correlating the fraction dose dissolved or dissolution rate versus fraction dose absorbed or absorption rate estimated by deconvolution. However, in most cases this requires that the absorption process is dissolution controlled. For extended-release products, there is a high probability of establishing an IVIVC. When an IVIVC cannot be established using deconvolution, convolution-based models should be used (level 3). Convolution-based approaches use models like the Advanced Compartmental Absorption and Transit models or other pharmacokinetic models to predict the oral performance of a dosage form. Biorelevant dissolution data are used in these models to predict the plasma-time curves. Such a prediction, if established by using the appropriate parameters, is a Level A correlation (14). Development of IVIVC/R requires data input including human pharmacokinetics, food effects, API properties $(B C S)$, and dosage-form information (excipient properties). For dosage forms having a positive food effect, FeSSIF should be used as the dissolution medium. For drug compounds largely absorbed in the intestine, simulated intestinal fluid offers high possibility of IVIVC. Computer tools can be used to develop the target profile for the biorelevant dissolution to achieve IVIVC.

A biorelevant dissolution, once developed, serves as the validated surrogate for in vivo performance. It can be of tremendous value in the rational design of formulation 


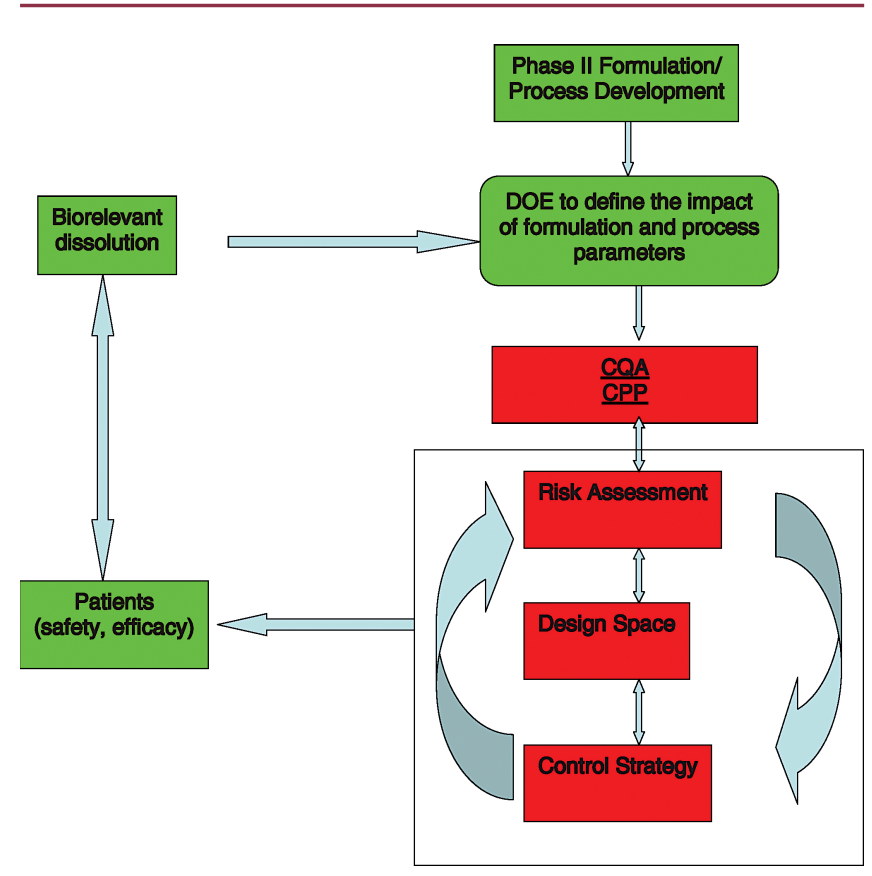

Figure 3. Biorelevant dissolution in bridging drug product to patient needs.

and process, from the early assessment of excipient impact on drug absorption to Design of Experiment (DOE) to identify CQA of the drug product. Compared with conventional development approaches, biorelevant dissolution is a powerful tool in achieving scientific understanding of the formulation and manufacturing processes, their relationship to product quality attributes, and ultimately their impact on the safety and efficacy of the product. The impact of any formulation changes on in vivo performance can be assessed, and formulation bridging may be established without the need for human studies.

Figure 3 reveals how biorelevant dissolution facilitates the Quality by Design approach to formulation and process development. With the challenges associated with IVIVC, especially for IR dosage forms, biorelevant dissolution can possibly be linked to in vivo performance via IVIVR. In such cases, this still presents a more indicative tool than the traditional QC method to guide formulation and process development. Biorelevant dissolution can be used in the Design of Experiments (DOE) to assess the impact of formulation properties and process parameters on product performance, which leads to the establishment of key quality attributes, the design space, and ultimately the overall control strategy. Through biorelevant dissolution, the link between product performance and patient needs is much more thoroughly understood.

\section{BIORELEVANT DISSOLUTION IN PHASE III CLINICAL DEVELOPMENT}

The primary objectives of Phase III development are to provide clinical supplies, generate formulation and manufacturing process knowledge for regulatory submission and approval, and prepare for a successful commercial product launch. To achieve these goals, it is necessary to optimize and finalize robust API and drug-product manufacturing processes, initiate long-term stability studies, and conduct pivotal bioequivalence and bioavailability (BE/BA) studies bridging Phase II and III clinical supplies and commercial product. Biorelevant dissolution can be used to facilitate the following activities:

- Enabling process control and real-time control strategy.

- Bridging Phase II and III formulations.

- Developing a more user-friendly dissolution test for QC purposes.

As in Phase II development, the biorelevant dissolution test can be a powerful tool in Phase III development to optimize and finalize the drug product formulation and process for commercial manufacture. Also, at Phase III development, control strategy and justification of specifications on critical quality attributes are established. If the mechanism of in vitro dissolution and the impact of product properties on dissolution are well understood from DOE studies at Phase II development, alternative in-process control tests that can directly measure such formulation properties (API and granule particle size measurement, disintegration test, tablet hardness, etc.) can be developed and correlated with the corresponding dissolution data. This presents opportunities for real-time release and process-control strategy, because product quality is controlled at line or on line during the manufacturing process instead of by the traditional QC dissolution test.

During Phase III development, formulation changes may involve API morphology, particle-size distribution, excipients, film coating, dose adjustment, or tablet shape. These changes may have an impact on the bioavailability of the active ingredient from the drug product. A biorelevant dissolution test should be used to assess the risk and impact of these changes on patients without additional biostudies, which will greatly increase the speed of drug development.

At Phase III and later stages of drug product life cycle, dissolution testing is an important quality control tool for monitoring batch-to-batch consistency or discriminating the impact of formulation or process changes on product performance. Such a quality control method should be simple, robust, and user-friendly. A biorelevant dissolution test often does not meet such criteria, and therefore, there should be an attempt to correlate the biorelevant dissolution test with a QC-friendly dissolution method. The hydrodynamics and medium of such a QC method should be selected to ensure batch-to-batch product consistency and to sufficiently discriminate the changes in product quality attributes as defined in Phase II development. Again, dissolution may not be needed 
or may be replaced by related techniques such as disintegration or another surrogate test (e.g., API particle size by NIR).

\section{REFERENCES}

1. Dressman, J. B.; Amidon, G. L.; Reppas, C.; Shah, V. P. Dissolution testing as a prognostic tool for oral drug absorption: immediate release dosage forms. Pharm. Res. 1998, 15 (1), 11-22.

2. Lipka, E.; Amidon, G. L. Setting bioequivalence requirements for drug development based on preclinical data: optimizing oral drug delivery systems. J. Controlled Release 1999, 62, 41-49.

3. Jantratid, E.; Janssen, N.; Reppas, C.; Dressman, J. B. Dissolution media simulating conditions in the proximal human gastrointestinal tract: an update. Pharm. Res. 2008, 25 (7), 1663-1676.

4. Vertzoni, M.; Dressman, J.; Butler, J.; Hempenstall, J.; Reppas, C. Simulation of fasting gastric conditions and its importance for the in vivo dissolution of lipophilic compounds. Eur. J. Pharm. Biopharm. 2005, 60 (3), 413-417.

5. Zangenberg, N. H.; Müllertz, A.; Kristensen, H. G.; Hovgaard, L. A dynamic in vitro lipolysis model. Il: Evaluation of the model. Eur. J. Pharm. Sci. 2001, 14, 237-244.

6. Garbacz, G.; Wedemeyer, R. S.; Nagel, S.; Giessmann, T.; Mönnikes, H.; Wilson, C. G.; Siegmund, W.; Weitschies, W. Irregular absorption profiles observed from diclofenac extended-release tablets can be predicted using a dissolution test apparatus that mimics in vivo physical stresses. Eur. J. Pharm. Biopharm. 2008, 70 (2), 421-428.

7. SUPAC-MR: Modified Release Solid Oral Dosage Forms: Scale-Up and Postapproval Changes: Chemistry, Manufacturing, and Controls; In Vitro Dissolution Testing and In Vivo Bioequivalence Documentation; Guidance for Industry; U.S. Department of Health and Human Services, Food and Drug Administration, Center for Drug Evaluation and Research (CDER), U.S. Government Printing Office: Washington, DC, 1997.

8. Dissolution Testing of Immediate Release Solid Oral Dosage Forms; Guidance for Industry; U.S. Department of Health and Human Services, Food and Drug Administration, Center for Drug Evaluation and Research (CDER), U.S. Government Printing Office: Washington, DC, 1997.

9. Katori, N.; Aoyagi, N.; Terao, T. Estimation of agitation intensity in the Gl tract in humans and dogs based on in vitro/in vivo correlation. Pharm. Res. 1995, 12 (2), 237-243.

10. D'Arcy, D. M.; Healy, A. M.; Corrigan, O. I. Towards determining appropriate hydrodynamic conditions for in vitro in vivo correlations using computational fluid dynamics. Eur. J. Pharm. Sci. 2009, 37 (3-4), 291-299.
11. Klein, S.; Rudolph, M. W.; Skalsky, B.; Petereit, H. U.; Dressman, J. B. Use of the BioDis to generate a physiologically relevant IVIVC. J. Controlled Release 2008, 130 (3), 216-219.

12. Fotaki, N.; Reppas, C. The flow-through cell methodology in the evaluation of intralumenal drug release characteristics. Dissolution Technol. 2005, $12(2), 17-21$.

13. Fotaki, N.; Aivaliotis, A.; Butler, J.; Dressman, J.; Fischbach, M.; Hempenstall, J.; Klein, S.; Reppas, C. A comparative study of different release apparatus in generating in vitro-in vivo correlations for extended release formulations. Eur. J. Pharm. Biopharm., in press.

14. Löbenberg, R.; Krämer, J.; Shah, V. P.; Amidon, G. L.; Dressman, J. B. Dissolution testing as a prognostic tool for oral drug absorption: dissolution behavior of glibenclamide. Pharm. Res. 2000, 17 (4), 439-444.

15. Sunesen, V. H.; Pedersen, B. L.; Kristensen, H. G.; Müllertz, A. In vivo in vitro correlations for a poorly soluble drug, danazol, using the flow-through dissolution method with biorelevant dissolution media. Eur. J. Pharm. Sci. 2005, 24 (4), 305-313.

16. Nicolaides, E.; Symillides, M.; Dressman, J. B.; Reppas, C. Biorelevant dissolution testing to predict the plasma profile of lipophilic drugs after oral administration. Pharm. Res. 2001, 18, 380-388.

17. Fotaki, N.; Symillides, M.; Reppas, C. Canine vs. in vitro data for predicting input profiles of L-sulpiride after oral administration. Eur. J. Pharm. Sci. 2005, 26 (3-4), 324-333.

18. Gribbon, P.; Sewing, A. High-throughput drug discovery: what can we expect from HTS? Drug Discov. Today 2005, 10 (1), 17-22.

19. Lipinski, C. A.; Lombardo, F.; Dominy, B. W.; Feeney, P. J. Experimental and computational approaches to estimate solubility and permeability in drug discovery and development settings. Adv. Drug Deliv. Rev. 2001, $46(1-3), 3-26$.

20. Gould, P. L. Salt selection for basic drugs. Int. J. Pharm. 1986, 33 (1-3), 201-217.

21. Kostewicz, E. S.; Wunderlich, M.; Brauns, U.; Becker, R.; Bock, T.; Dressman, J. B. Predicting the precipitation of poorly soluble weak bases upon entry in the small intestine. J. Pharm. Pharmacol. 2003, 56 (1), 43-51.

22. Dressman, J. B.; Amidon, G. L.; Fleisher, D. Absorption potential: estimating the fraction absorbed for orally administered compounds. J. Pharm. Sci. 1985, 74 (5), 588-589.

23. Lui, C. Y.; Amidon, G. L.; Berardi, R. R.; Fleisher, D.; Youngberg, C.; Dressman, J. B. Comparison of gastrointestinal $\mathrm{pH}$ in dogs and humans: implications on the use of the beagle dog as a model for oral absorption in humans. J. Pharm. Sci. 1986, 75 (3), 271-274.

24. Dressman, J. B.; Yamada, K. Animal Models for Oral Drug Absorption. In Pharmaceutical Bioequivalence; Welling, P. G., Tse, F. L. S, Dighe, S. V., Eds.; Drugs in 
the Pharmaceutical Sciences; Marcel Dekker, Inc.:

New York, 1991; Vol. 48, pp 235-266.

25. Dokoumetzidis, A.; Kalantzi, L.; Fotaki, N. Predictive models for oral drug absorption: from in silico methods to integrated dynamical models. Expert Opin.

Drug Metab. Toxicol. 2007, 3 (4), 491-505.
26. Persson, E. M.; Gustafsson, A. S.; Carlsson, A. S.; Nilsson, R. G.; Knutson, L.; Forsell, P.; Hanisch, G.; Lennernaes, $\mathrm{H}$.; Abrahamsson, B. The Effects of Food on the Dissolution of Poorly Soluble Drugs in Human and in Model Small Intestinal Fluids. Pharm. Res. 2005, 22 (12), 2141-2151.

\section{Basic Dissolution Testing: AAPS Short Course on CD-ROM}

About the Course:

Dissolution testing is a critical test for measuring the performance of a drug product. The dissolution test has been a relatively new analytical technique with equipment modifications and improvements spanning ably in the last 10 years. It is a quality control tool and an aid to formulation development. Dissolution testing measures change on stability, and is used to establish an in-vitro and in-vivo correlation for some products.

\section{Course Contents:}

- General Overview

\section{- Equipment}

Distance Learning

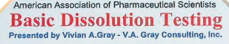

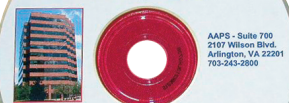

Sources of Error During

Calibration and Sample Testing

- Utility of Dissolution Testing

- Selecting Test Conditions and Method Development

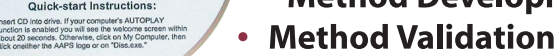

- Role of USP in Dissolution Testing

- FDA Role in Dissolution and

Guidances, Other Regulatory

Guidances, and Additional Resources

About the Presenter:

Vivian A. Gray was the Head of the Dissolution Group in the Analytical Research and Development Section of DuPont Pharmaceuticals Company in Wilmington, Delaware when this presentation was prepared in 2000

Prior to joining DuPont Pharmaceuticals Company, she was with the United
States Pharmacopeia in Rockville, Maryland. Vivian has spent the last 30 years involved in all aspects of dissolution testing, including regulatory issues, calibration, dissolution method development and validation, and evaluating new dissolution technology. In 2002, she formed her own consulting company in dissolution testing and related areas. The company name is V. A. Gray, Consulting, Inc., www.vagrayconsulting.com, located in Hockessin, DE. She is now Managing Director of Dissolution Technologies, at www.dissolutiontech.com

\section{Who Should Participate:}

This CD-ROM Short Course is suitable for anyone who must need to understand dissolution and how dissolution tests are performed, including those involved in formulation, QC, R\&D, metrology, and regulation.

\section{Technical Platform and Requirements:}

This two-hour course is contained within a standard CD-ROM Requirements include a 486 or faster PC running Windows 9x, a CD-ROM player, sound card, and speakers. Included on the disk is a copy of PowerPoint 97 Viewer, which is required to view the course content, and which must be installed in the default directory in order to properly utilize the menu-driven program.

Price:

$\$ 180.00$ domestic, $\$ 200.00$ outside USA. Credit card payment available.

For complete ordering information go online to

www.dissolutiontech.com or contact:

Dissolution Technologies

9 Yorkridge Trail, Hockessin, DE 19707

Phone-302-235-0621, Fax-443-946-1264

Email:vgray@ dissolutiontech.com 